\title{
ON THE ERGODIC THEOREM
}

\author{
SHIGERU TSURUMI
}

(Received April 15, 1954)

1. Introduction. Let $(X, \mathfrak{B}, m)$ be a measure space such that $X$ is a set, $\mathfrak{B}$ is a Borel field of subsets of $X$, and $m$ is a $\sigma$-finite measure defined on $\mathfrak{B}$. A single valued transformation $T$ of $X$ into itself is called measurable if the inverse transformation $T^{-1}$ sends every set of $\mathfrak{B}$ to a set of $\mathfrak{B}$. The measurable transformation $T$ is called non-singular (with respect to $m$ ) if $A \in \mathfrak{B}$ and $m(A)=0$ imply $m\left(T^{-1} A\right)=0$. Throughout this paper it is assumed that all sets under consideration are in $\mathfrak{B}$ and the transformation $T$ is measurablea nd non-singular. A measure $\mu$ defined on $\mathfrak{B}$ is said to be invariant under $T$ (or $T$ is said to be measure-preserving with respect to $\mu$ ) if $\mu\left(T^{-1} A\right)=$ $\mu(A)$ for every set $A$. Two measures $\lambda$ and $\mu$ defined Ion $\mid \mathfrak{B}$ are called equivalent if $\lambda(A)=0$ implies $\mu(A)=0$ and the converse. A set $A$ is called an invariant set if $m\left(T^{-1} A-A\right)+m\left(A-T^{-1} A\right)=0$.

We define the following statements.

(I) There exists a constant $K$ such that

$$
0<\lim \sup _{n} \frac{1}{n} \sum_{i=0}^{n-1} m\left(T^{-i} A\right) \leqq K \cdot m(A)
$$

for every set $A$ of positive measure.

(I') There exists a constant $K$ such that

$$
\lim _{n} \sup \frac{1}{n} \sum_{i=0}^{n-1} m\left(T^{-i} A\right) \leqq K \cdot m(A)
$$

for every set $A$.

(II) There exists a sequence of sets $\left\{X_{j}\right\}$ and a constant $K$ such that

$$
X_{1} \subset X_{2} \subset \ldots, X=\bigcup_{j=1}^{\infty} X_{j}, m\left(X_{j}\right)<\infty(j=1,2, \ldots),
$$

and

$$
0<\sup _{j} \lim \sup _{n} \frac{1}{n} \sum_{i=0}^{n-1} m\left(X_{j} \cap T^{-i} A\right) \leqq K \cdot m(A)
$$

for every set $A$ of positive measure.

(II') There exists a sequence of sets $\left\{X_{j}\right\}$ and a constant $K$ such that

and

$$
X_{1} \subset X_{2} \subset \ldots, X=\bigcup_{j=1}^{\infty} X_{j}, m\left(X_{j}\right)<\infty(j=1,2, \ldots),
$$

$$
\sup _{j} \lim _{n} \sup \frac{1}{n} \sum_{i=0}^{n-1} m\left(X_{j} \cap T^{-i} A\right) \leqq K \cdot m(A)
$$


for every set $A$.

(B) For any function $f \in L(X, \mathfrak{B}, \boldsymbol{m})^{1)}$ the limit

$$
\widetilde{f}(x)=\lim _{n} \frac{1}{n} \sum_{i=0}^{n-1} f\left(T^{i} x\right)
$$

exists almost everywhere on $X$ and $\widetilde{f} \in L(X, \mathfrak{B}, m)$.

In the following, we use the notation $\widetilde{f}(g$ etc. $)$ which denotes the limit function of the means $\frac{1}{n} \sum_{i=0}^{n-1} f\left(T^{i} x\right)\left(\frac{1}{n} \sum_{i=0}^{n-1} g\left(T^{i} x\right)\right.$ etc. $)$ in case the limit is well defined.

In case $\boldsymbol{m}$ is finite, N. Dunford and D.S. Miller [1] ${ }^{2)}$ have given in their joint paper a necessary and sufficient condition ${ }^{3)}$ that Neumann's ergodic theorem holds, and as its consequence led the statement $(B)$ from this condition. Hereafter F.Riesz [3] has given another proof of the latter and proved that, even if $m$ is not finite, the above condition with a certain additional restriction implies $(B)$ (see Corollary of Theorem 1 in $\S 2$ ). Recently C. Ryll-Nardzewski [4] has shown that the statement (II'), which is weaker than (II) formulated by S. Hartman, is equivalent to $(B)$ and that, in case $\boldsymbol{m}$ is finite, the statements (II), (II') and $(B)$ are equivalent to each other. However a part of the former is not quite right in case $m$ is not finite. In fact we can construct a $\sigma$-finite (but not finite) measure space and a transformation for which $\left(\mathrm{II}^{\prime}\right)$ holds and $(B)$ does not hold (see Example 1 in $\$ 3)$.

The main purpose of this paper is to work out that each of (I) and (II) implies $(B)$, and $\left(\mathrm{II}^{\prime}\right)$ does not necessarily imply $(B)$.

2. Generalization of Birkhoff's ergodic theorem. Let $\Delta(A)=\Delta(A$, $\left.\left\{A_{k}\right\}_{k=1,2}, \ldots\right)$ denote a decomposition of the set $A$ such that

$$
A=\bigcup_{k=1}^{\infty} A_{k}, A_{k} \cap A_{l}=0(k \neq l) \text {. }
$$

Let us put for every set $A$

$$
\alpha(A)=\sup _{(A)} \sum_{(A: k)} \lim \sup _{n} \frac{1}{n} \sum_{i=0}^{n-1} m\left(T^{-i} A_{k}\right)
$$

where $\sup _{(A)}$ denotes the supremum for all decompositions $\Delta(A)$ of $\mid A$ and $\sum_{(A: k)}$ means to sum up with respect to all sets $A_{i x}$ 's of the Idecomposition

1) The notation $L(X, \mathfrak{B}, m)$ denotes the class of all integrable functions with respect to the measure space $(X, \mathfrak{B}, m)$.

2) Numbers in square brackets refer to the references at the end of this paper. set $A$

3) The condition reads as follows : there exists a constant $K$ such that for any

$$
\frac{1}{n} \sum_{i=0}^{n-1} m\left(T^{-i} A\right) \leqq \dot{K} \cdot m(A) \quad(n=1,2, \ldots \ldots) .
$$


$\Delta(A)=\Delta\left(A, \quad\left\{A_{k}\right\}_{k=1,2, \ldots)}\right.$.

LEMMA 1. If the statement $\left(\mathrm{I}^{\prime}\right)$ holds, the non-ne gative set function $\alpha$ defined by (1) has the following properties:

(i) $\alpha$ is finitely additive;

(ii) $\alpha(A) \leqq \alpha\left(T^{-1} A\right)$ for every set $A$ of finite measure;

(iii) $\alpha(A) \leqq K \cdot m(A)$ for every set $A$;

(iv) $\alpha(A) \geqq \limsup _{n} \frac{1}{n} \sum_{i=0}^{n-1} m\left(T^{-i} A\right)$ for every set $A$.

Proof. Proof of (i): Let us suppose that

$$
A=\bigcup_{j=1}^{N} A^{j}, \quad A^{i} \cap A^{j}=0(i \neq j) .
$$

Let $\varepsilon$ be any positive number. Then, for each $j$, there exists a decomposition $\Delta\left(A^{j},\left\{A_{k}^{\jmath}\right\}_{k=1,2}, \ldots\right)$ such that

$$
\alpha\left(A^{j}\right)-\frac{\varepsilon}{N}<\sum_{(A j: k)} \lim \sup _{n} \frac{1}{n} \sum_{i=0}^{n-1} m\left(T^{-i} A_{k}^{j}\right) .
$$

Combining all $\Delta\left(A^{j}\right)^{\text {'s }}$ we get a decomposition $\Delta\left(A,\left\{A_{k}^{j}\right\}_{k=1,2}, \ldots ; j=1,2\right.$, $\ldots, N)$, so that it follows

$$
\begin{gathered}
\sum_{j=1}^{N} \alpha\left(A^{j}\right)-\varepsilon<\sum_{j=1}^{N} \sum_{(A ;: k)} \lim \sup _{n} \frac{1}{n} \sum_{i=0}^{n-1} m\left(T^{-i} A_{k}^{j}\right) \\
=\sum_{(A: k, j)} \lim \sup _{n} \frac{1}{n} \sum_{i=0}^{n-1} m\left(T^{-i} A_{k}^{j}\right) \leqq \alpha(A) .
\end{gathered}
$$

On the other hand, there exists a decomposition $\Delta\left(A,\left\{A_{k}\right\}_{k=1,2,} \ldots\right)$ such that

$$
\alpha(A)-\varepsilon<\sum_{(A: k)} \lim \sup _{n} \frac{1}{n} \sum_{i=0}^{n-1} m^{\prime}\left(T^{-i} A_{k}\right) .
$$

Let us put

$$
A_{k}^{j}=A_{k} \cap A^{j}(k=1,2, \ldots ; j=1,2, \ldots, N) .
$$

Then, for each $j$, the collection of sets $\left\{A_{k}^{j}\right\}_{k=1,2 \ldots} \ldots$ gives a decomposition $\Delta\left(A^{j},\left\{A_{k}^{j}\right\}_{k=1,2,}, \ldots\right)$, so that we have

$$
\begin{aligned}
\sum_{(A: k)} \lim \sup _{n} \frac{1}{n} \sum_{i=0}^{n-1} m\left(T^{-i} A_{k}\right) \leqq \sum_{(A: k, j)} \lim \sup _{n} \frac{1}{n} \sum_{i=0}^{n-1} m\left(T^{-i} A_{k}^{j}\right) \\
=\sum_{j=1} \sum_{(A j: k)} \lim \sup _{n} \frac{1}{n} \sum_{i=0}^{n-1} m\left(T^{-i} A_{k}^{j}\right) \leqq \sum_{j=1}^{N} \alpha\left(A^{j}\right) .
\end{aligned}
$$

Thus by (3) and (4) we have

$$
\alpha(A)-\varepsilon<\sum_{j=\mathrm{P}^{\mathrm{p}}}^{N} \alpha\left(A^{j}\right) .
$$

Since $\varepsilon$ is arbitrary, (2) and (5) imply 


$$
\alpha(A)=\sum_{j=1}^{N} \alpha\left(A^{j}\right) .
$$

Hence it follows that $\alpha$ is finitely additive.

Proof of (ii): If $A$ is a set of finite measure, then for each $i$ the set $T^{-i} A$ is of finite measure. Further, to a decomposition $\Delta\left(A,\left\{A_{k}\right\}_{k=1,2,}, \ldots\right)$ there corresponds a decomposition $\Delta\left(T^{-1} A,\left\{T^{-1} A_{k}\right\}_{k=1,2,} \ldots\right)$. Hence we can easily show the property (ii).

The properties (iii) and (iv) are the immediate consequences of the definition of $\alpha$.

C Ryll-Nardzewski [4] has proved the following

LEMMA 2. Let $\left(X^{\prime}, \mathfrak{B}^{\prime}, \mu\right)$ be a measure space, and let $\widetilde{T}$ be a transformation of $L\left(X^{\prime}, \mathfrak{B}^{\prime}, \mu\right)$ into itself which has the following properties:

(i) if $f(x)=g(x)$ almost everywhere $(\mu), \widetilde{T} f(x)=\widetilde{T} g(x)$ almost everywhere $(\mu)$;

(ii) $\widetilde{T}$ is additive and homogeneous;

(iii) if $f(x)$ is positive almost everywhere $(\mu), \widetilde{T} f(x)$ is also. Then $\widetilde{T}$ is a linear operator of $L\left(X^{\prime}, \mathfrak{B}^{\prime}, \mu\right)$ into itself.

We shall now prove the following theorem which is a generalization of Birkhoff's ergodic theorem.

THEOREM 1. The statement (I) implies the statement $(B)$, but the converse is not true. other.

If $m$ is finite, three statements $(I),\left(I^{\prime}\right)$ and $(B)$ are equivalent to each

Proof. $(\mathrm{I}) \rightarrow(B)$ : We put for any set $A$ of finite measure

$$
\beta(A)=\lim _{n} \alpha\left(T^{-n} A\right) .
$$

This definition is justified by (ii) of Lemma 1 .

Let $B$ be a fixed set of finite measure, then the non-negative set function ,$\beta(A \cap B)$ of variable $A$ has the following properties :

(i) $\beta(A \cap B) \leqq K^{2} \cdot m(A \cap B)$ for every set $A$;

(ii) $\beta(A \cap B)$ is completely additive as the set function of $A$;

(iii) $\beta\left[T^{-1}(A \cap B)\right]=\beta(A \cap B)$ for every set $A$;

(iv) $\beta(A \cap B) \geqq \alpha(A \cap B) \geqq \lim \sup _{n} \frac{1}{n} \sum_{i=0}^{n-1} m\left[T^{-1}(A \cap B)\right]$ for levery set $A$. set $A$

Proof of (i): From (ii), (iii) of Lemma 1 and (I), it follows that for any

$$
\begin{aligned}
\beta(A \cap B)= & \lim _{n} \alpha\left[T^{-n}(A \cap B)\right] \\
& \lim \frac{1}{n} \sum_{i=0}^{n-1} \alpha\left[T^{-i}(A \cap B)\right]
\end{aligned}
$$




$$
\leqq \lim _{n} \sup \frac{1}{n} \sum_{i=0}^{n-1} K \cdot m\left[T^{-i}(A \cap B)\right] \leqq K^{2} \cdot m(A \cap B),
$$

which is the required.

Proof of (ii): From (i) of Lemma 1 it follows that $\beta(A \cap B)$ is finitely additive as the set function of $A$.

Let $\left\{A_{n}\right\}$ be any sequence of sets such that

$$
A_{1} \supset A_{2} \supset \ldots, \bigcap_{n=1}^{\infty} A_{n}=0 .
$$

Since $B$ is of finite measure, $m\left(A_{n} \cap B\right)$ tends to zero with $1 / n$, so that, from (i), $\beta\left(A_{n} \cap B\right)$ tends to zero with $1 / n$. Thus $\beta(A \cap B)$ is completely additive.

The properties (iii) and (iv) follows evidently from the definition of $\beta$ and (iv) of Lemma 1.

Now we choose a sequence of sets $\left\{Y_{k}\right\}$ such that

$$
\mathrm{X}=\bigcup_{k=1}^{\infty} Y_{k}, Y_{k} \cap Y_{l}=\mathrm{U}(k \neq l), m\left(Y_{k}\right)<\infty(k=1,2, \ldots) .
$$

Let us put for each set $A$

$$
\gamma(A)=\sum_{k=1}^{\infty} \beta\left(A \cap Y_{k}\right)
$$

Then the non-negative set function $\gamma$ has the following properties :

(v) $\gamma(A) \leqq K^{2} \cdot m(A)$ for every set $A$;

(vi) $\gamma$ is an invariant measure on $\mathfrak{B}$;

(vii) $\gamma$ is equivalent to $m$;

(viii) $\gamma(A) \geqq m(A)$ for any invariant set $A$.

The property (v) is the immediate consequence of (i) and definition of $\gamma$.

Proof of (vi): From (ii) it is evident that $\gamma$ is a measure on $\mathfrak{B}$, so that we shall prove only that $\gamma$ is invariant under $T$. Since $Y_{k}$ 's are of finite measure and hence, from (I), $T^{-1} Y_{k}$ 's are also, it follows, from (iii), that for any set $A$

$$
\begin{aligned}
\gamma(A) & =\sum_{k=1}^{\infty} \beta\left(A \cap Y_{k}\right)=\sum_{k=1}^{\infty} \beta\left[T^{-1}\left(A \cap Y_{k}\right)\right] \\
& =\sum_{k=1}^{\infty} \sum_{l=1}^{\infty} \beta\left(T^{-1} A \cap T^{-1} Y_{k} \cap Y_{l}\right) \\
& =\sum_{l=1}^{\infty} \sum_{k=1}^{\infty} \beta\left(T^{-1} A \cap T^{-1} Y_{k} \cap Y_{l}\right) \\
& =\sum_{l=1}^{\infty} \beta\left(T^{-1} A \cap Y_{l}\right)=\gamma\left(T^{-1} A\right) .
\end{aligned}
$$

Proof of (vii): It is clear that $m(A)=0$ implies $\gamma(A)=0$, so that we shall prove the converse. 
Let $A$ be any set of positive measure. Then there is a set $Y_{k_{0}}$ such that the set $A \cap Y_{k_{0}}$ is of positive measure. From (iv) and (I) it follows

$$
\begin{aligned}
\gamma(A) & =\sum_{k=1}^{\infty} \beta\left(A \cap Y_{k}\right) \geqq \beta\left(A \cap Y_{k_{0}}\right) \\
& \geqq \lim \sup _{n} \frac{1}{n} \sum_{i=0}^{n-1} m\left[T^{-i}\left(A \cap Y_{k_{0}}\right)\right]>0 .
\end{aligned}
$$

Proof of (viii): Let $A$ be an arbitrary fixed invariant set. If $A$ is not of finite $\gamma$-measure, it holds obviously

$$
\gamma(A) \geqq m(A),
$$

so that we shall consider the case where $A$ is of finite $\gamma$-measure. Then from (vi) and (vii) it follows that $\gamma$ is a finite invariant measure equivalent to $m$ as the measure on $\mathfrak{B}_{A}^{4}$. By Birkhoff's ergodic theorem, for each $f \in L(A$, $\left.\mathfrak{B}_{A}, \gamma\right)$ the limit function $\widetilde{f}(x)$ of the means $\frac{1}{n} \sum_{i=0}^{n-1} f\left(T^{i} x\right)$ is defined almost everywhere $(\gamma)$ on $A$ and $\widetilde{f} \in L\left(A, \mathfrak{B}_{A}, \gamma\right)$. Since $m$ is equivalent to $\gamma$, the function $\widetilde{f}$ is also defined almost everywhere $(m)$ on $A$. Let $\widetilde{T}$ denotes the transformation of $L\left(A, \mathfrak{B}_{A}, \gamma\right)$ into itself defined by

$$
\widetilde{T f}=\widetilde{f}
$$

for each $\widetilde{f} \in L\left(A, \mathfrak{B}_{A}, \gamma\right)$. Then $\widetilde{T}$ has the properties (i), (ii) and (iii) of Lemma 2 , so that $\widetilde{T}$ is a linear operator of $L\left(A, \mathfrak{B}_{A}, \gamma\right)$ into itself. Let us now suppose that $\Delta\left(B,\left\{B_{k}\right\}_{k=1,2, \ldots)}\right.$ is a decomposition of any subset $B$ of $A$, and let $\phi_{B}$ and $\phi_{B_{k}}$ 's be the characteristic functions of $B$ and $B_{k}$ 's, respectively $^{5}$. Then it holds

$$
\int_{A}\left|\varphi_{B}(x)-\sum_{k=1}^{N} \varphi_{B_{k}}(x)\right| d \gamma \rightarrow 0(N \rightarrow \infty)
$$

so that from the linearity of $\widetilde{T}$ it follows

$$
\int_{A}\left|\widetilde{\boldsymbol{\phi}}_{B}(x)-\sum_{k=1}^{N}{\widetilde{\phi_{B}}}_{B_{k}}(x)\right| d \gamma=\int_{A}\left|\widetilde{T}\left(\phi_{B}(x)-\sum_{k=1}^{N} \phi_{B_{k}}(x)\right)\right| d \gamma \rightarrow 0(N \rightarrow \infty) .
$$

Since $\sum_{k=1}^{N} \widetilde{\mathcal{P}}_{B_{k}}(x)$ is monotone-increasing as $N$ increases, we have that

$$
\sum_{k=1}^{\infty}{\widetilde{\boldsymbol{\rho}_{B}}}_{B_{k}}(x)=\widetilde{\boldsymbol{\varphi}}_{B}(x)
$$

almost everywhere $(\gamma)$ on $A$ and hence almost everywhere $(m)$ on $A$.

Therefore, by Fatou's lemma we have

4) The notation $\mathfrak{B}_{A}$ denotes the Borel field relative to the set $A$ (that is, $\mathfrak{B}_{A}$ is consisted of all subsets of $A$ ).

5) In the following we employ the notations $\phi_{A}, \phi_{B}$ etc. for the characteristic functions. 


$$
\begin{aligned}
& \gamma(A)=\sum_{l=1}^{\infty} \beta\left(A \cap Y_{l}\right) \geqq \sum_{l=1}^{\infty} \alpha\left(A \cap Y_{l}\right) \\
& =\sum_{l=1}^{\infty} \sup _{\left(A \cap Y_{l}\right)} \sum_{\left(A \cap Y_{l} k^{k}\right)} \lim \sup _{n} \frac{1}{n} \sum_{i=0}^{n-1} m_{l}\left[T^{-i}\left(A \cap Y_{l}\right)_{k}\right] \\
& =\sum_{l=1}^{\infty} \sup _{\left(A \cap Y_{l}\right)} \sum_{\left(A \cap Y_{l}: k\right)} \limsup _{n} \frac{1}{n} \sum_{i=0}^{n-1} \int_{A} \varphi_{\left(A \cap Y_{l} k\right.}\left(T^{i} x\right) d m \\
& \geqq \sum_{l=1}^{\infty} \sup _{\left(A \cap Y_{l}\right)} \sum_{\left(A \cap Y_{l}: k\right)} \int_{A} \lim \inf _{n} \frac{1}{n} \sum_{i=0}^{n}-1 \\
& =\sum_{l=1}^{\infty} \sup _{\left(A \cap Y_{l}\right.} \sum_{\left(A \cap Y_{l}: k\right)} \int_{A} \widetilde{\varphi}_{\left(A \cap Y_{l}\right)_{k}}(x) d m \\
& =\sum_{l=1}^{\infty} \int_{A} \widetilde{\phi}_{A \cap Y_{l}}(x) d m=\int_{A} \widetilde{\varphi}_{A}(x) d m=\int_{A} 1 d m=m(A) .
\end{aligned}
$$

Thus the proof of (viii) is complete.

Next let us suppose $f \in L(X, \mathfrak{B}, m)$, then by (v) we have $f \in L(X, \mathfrak{B}, \gamma)$. Hence from Birkhoff's ergodic theorem it follows that the limit

$$
\widetilde{f}(x)=\lim _{n} \frac{1}{n} \sum_{i=0}^{n-1} f\left(T^{i} x\right)
$$

exists almost everywhere $(\gamma)$ and then almost everywhere $(m)$ on account of (vii), and furthermore $\widetilde{f} \in L(X, \mathfrak{B}, \gamma)$. Since the limit function $\widetilde{f}$ is an invariant function, it follows that, for any pair of real numbers $a$ and $b$, the set $\{x$; $a \leqq \widetilde{f}(x)<b\}$ is an invariant set. Hence, from $\widetilde{f} \in L(X, \mathfrak{R}, \gamma)$, (viii', and the definition of the integral, we have easily $\widetilde{f} \in L(X, \mathfrak{B}, m)$. Thus it was proved that (I) implies $(B)$. (I).

It will be shown by Example 2 in $\S 3$ that $(B)$ does not necessarily imply

Next we shall prove that, in case $m$ is finite, the statements (I), (I') and $(B)$ are equivalent to each other. We have already proved that (I) implies $(B)$, so that it remains to prove that $(B)$ implies $\left(I^{\prime}\right)$ and $\left(I^{\prime}\right)$ implies (I).

$\left.(B) \rightarrow\left(I^{\prime}\right)^{6}\right)$ : Since $m$ is finite, we have, by Lebesgue's convergence theorem, $(B)$ and Lemma 2, that there exists a constant $K$ such that for any set $A$,

$$
\lim \sup _{n} \frac{1}{n} \sum_{i=0}^{n-} m\left(T^{-i} A\right)=\lim _{n} \sup \frac{1}{n} \sum_{i=0}^{n-1} \int_{X} \phi_{A}\left(T^{i} x\right) d m
$$

6) The proof of this part is due to Ryll-Nardzewski [4]. 


$$
\leqq \int_{X} \lim \sup _{n} \frac{1}{n} \sum_{i=0}^{n-1} \phi_{.1}\left(T^{i} x\right) d m=\int_{X} \widetilde{\boldsymbol{\phi}}_{A}(x) d m \leqq K \int_{X} \phi_{A}(x) d m=K \cdot m(A) .
$$

which is the required.

$\left(\mathrm{I}^{\prime}\right) \rightarrow(\mathrm{I})$ : Since $m$ is finite, we can show that for any set $A$

$$
\gamma(A)=\beta(A)=\lim _{n} \alpha\left(T^{-n} A\right) .
$$

Let us now suppose that there exists a set $A$ such that

$$
m(A)>0, \quad \lim _{n} \sup \frac{1}{n} \sum_{i=0}^{n-1} m\left(T^{-i} A\right)=0 .
$$

Then it is easy to see that for each positive integer $j$

$$
\lim \sup _{n} \frac{1}{n} \sum_{i=0}^{n-1} m\left[T^{-i}\left(T^{-i} A\right)\right]=\lim _{n} \sup \frac{1}{n} \sum_{i=0}^{n-1} m\left(T^{-i} A\right)=0 .
$$

Hence from the definition of $\alpha$ it follows

$$
\alpha\left(T^{-n} A\right)=0(n=0,1,2, \ldots) .
$$

Let us put

$$
\widetilde{A}=\bigcup_{i=0}^{\infty} T^{-i} A
$$

Since, in the present case, $\gamma$ is a finite invariant measure and $\widetilde{A} \supset T^{-1} \widetilde{A}$, it follows that $\widetilde{A}$ is an invariant set. Thus we have

$$
\gamma(\widetilde{A}) \geqq m(\widetilde{A}) \geqq m(A)>0 .
$$

On the other hand, from (6) and (7), it follows

$$
\gamma(\widetilde{A}) \leqq \sum_{i=0}^{\infty} \gamma\left(T^{-i} A\right)=\sum_{i=0}^{\infty} \gamma(A)=0 .
$$

This contradiction shows that if $A$ is any set of positive measure, it follows

$$
\lim \sup _{n} \frac{1}{n} \sum_{i=0}^{n-1} m\left(T^{-i} A\right)>0 .
$$

Hence, from $\left(\mathrm{I}^{\prime}\right)$ and the above inequality we get (I).

Thus Theorem 1 is now completely proved.

REMARK 1. The statement (I) implies that for any set $A$ of positive measure

$$
\liminf _{n} \frac{1}{n} \sum_{i=0}^{n-1} m\left(\mathrm{~T}^{-i} A\right)>0 .
$$

In fact, let $A$ be any set of positive measure, then we can choose a set $Y$ such that $0<m(A \cap Y)<\infty$. Then it is easy to see that for each positive integer $j$

$$
\lim _{n} \sup \frac{1}{n} \sum_{i=0}^{n-1} m\left[T^{-i}(A \cap Y)\right]
$$




$$
=\lim _{n} \sup \frac{1}{n} \sum_{i=0}^{n-1} m\left[T^{-i}\left(T^{-j}(A \cap Y)\right)\right] \leqq K \cdot m\left[T^{-j}(A \cap Y)\right] .
$$

Thus we have

$$
\lim \sup _{n} \frac{1}{n} \sum_{i=0}^{n-1} m\left[T^{-i}(A \cap Y)\right] \leqq K \cdot \inf _{j} m\left[T^{-j}(A \cap Y)\right]
$$

so that

$$
\begin{aligned}
& \lim _{n} \inf \frac{1}{n} \sum_{i=0}^{n-1} m\left(T^{-i} A\right) \geqq \inf _{,} m\left[\left(T^{-i}(A \cap Y)\right]\right. \\
\geqq & \frac{1}{K} \cdot \lim _{n} \sup \frac{1}{n} \sum_{i=0}^{n-1} m\left[T^{-i}(A \cap Y)\right]>0 .
\end{aligned}
$$

By the above remark, the proof of Theorem 1 may be considerably simplified, but our method of proof of Theorem 1 enables us to prove Theorem 2.

REMARK. 2. The question of whether $\left(I^{\prime}\right)$ implies $(B)$ or not is still open.

The following result due to F.Riesz [3] follows immediately from Theorem 1.

COROLLARY. If there exist two positive constants $K_{1}$ and $K_{2}$ such that for any set $A$

$$
K_{1} \cdot m(A) \leqq \frac{1}{n} \sum_{i=0}^{n-1} m\left(T^{-i} A\right) \leqq K_{2} \cdot m(A)(n=1,2, \ldots),
$$

then the statement $(B)$ holds.

Next we shall state the theorem which is a modefication of Ryll-Nardzewski's theorem [4].

THEOREM 2. The statements (II) and (B) imply the statements (B) and (II') respectively, but the converses are not true.

PRoof. $\left(\mathrm{II}^{\prime}\right) \rightarrow(B)$ : This implication is proved similarly as Lemma 1 and Theorem 1, so that we shall sketch the proof.

With respect to the sets $X_{j}$ 's in (II), we put

$$
\alpha^{*}(A)=\sup _{j} \sup _{(A)} \sum_{(A k)} \lim \sup _{n} \frac{1}{n} \sum_{i=0}^{n-1} m\left(X_{j} \cap T^{-i} A_{k}\right)
$$

for any set $A$. Then the non-negative set function $\alpha^{*}$ has the following properties :

(i) $\alpha *$ is finitely additive;

(ii) $\alpha^{*}(A) \leqq \alpha^{*}\left(T^{-1} A\right)$ for every set $A$;

(iii) $\alpha^{*}(A) \leqq K \cdot m(A)$ for every set $A$;

(vi) $\alpha *(A) \geqq \sup _{j} \lim \sup _{n} \frac{1}{n} \sum_{i=0}^{n-1} m\left(X_{j} \cap T^{-i} A\right)$ for every set $A$.

We put further for any set $A$ 


$$
\beta^{*}(A)=\lim _{n} \alpha *\left(T^{-n} A\right) .
$$

For any fixed set $B$ of finite measure, the non-negative set function $\beta^{*}(A \cap$ $B$ ) of variable $A$ has the following properties:

(v) $\beta^{*}(A \cap B) \leqq K^{2} \cdot m(A \cap B)$ for every set $A$;

(vi) $\beta^{\times}(A \cap B)$ is completely additive as the set function of $A$;

(vii) $\beta^{*}\left[T^{-1}(A \cap B)\right]=\beta *(A \cap B)$ for every set $A$;

(viii) $\beta^{\times}(A \cap B) \geqq \sup _{j} \lim _{n} \sup _{n} \frac{1}{n} \sum_{i=0}^{n-1} m\left[X, \cap T^{-i}(A \cap B)\right]$ for every set $A$.

Finally we put for any set $A$

$$
\gamma^{*}(A)=\lim _{j} \beta^{*}\left(A \cap X_{j}\right)
$$

Then the non-negative set function $\gamma^{*}$ has the following properties :

(ix) $\gamma^{*}(A) \leqq K^{2} \cdot m(A)$ for every set $A$;

(x) $\gamma^{*}$ is an invariant measure on $\mathfrak{B}$;

(xi) $\gamma^{*}$ is equivalent to $m$;

(xii) $\gamma^{*}(A) \geqq m(A)$ for any invariant set $A$.

From the properties (ix)-(xii) of $\gamma^{*}$, it is easy to see that (II) implies $(B)$.

$(B) \rightarrow\left(\mathrm{II}^{\prime}\right)^{7)}$ : From $(B)$ and Lemma 2 there exists a constant $K$ such that for any sets $Y$ and $A$ of finite measure

$$
\begin{aligned}
& \lim \sup _{n} \frac{1}{n} \sum_{i=1}^{n-1} m\left(Y \cap T^{-i} A\right)=\lim \sup _{n} \frac{1}{n} \sum_{i=0}^{n-1} \int_{Y} \phi_{A}\left(T^{i} x\right) d m \\
& \leqq \int_{Y} \lim \sup _{n} \frac{1}{n} \sum_{i=0}^{n-1} \phi_{A}\left(T^{i} x\right) d m=\int_{Y} \widetilde{\phi}_{A}(x) d m \leqq \int_{X} \widetilde{\phi}_{A}(x) d m \\
& \leqq K \int_{X} \phi_{A}(x) d m=K \cdot m(A)
\end{aligned}
$$

which implies $\left(\mathrm{II}^{\prime}\right)$.

It will be shown by Example 1 in $\S 3$ that $(B)$ and $\left(\mathrm{II} 1^{\prime}\right)$ do not necessarily imply (II) and $(B)$, respectively.

By use of Theorem 2 we shall prove the following two theorems.

THEOREM 3. If there exists a finite invariant measure equivalent to $m$, then the statements (II), (II') and (B) are equivalent to each other.

The assumption of the theorem cannot be omitted.

Proof. Theorem 2 shows that (II) implies $(B)$ and $(B)$ Implies (II'), so that for the present purpose it is sufficient to prove that (II') implies (II).

Let $A$ ibe any set of positive measure, $\mu$ a finite invariant measure equivalent to $m$, and $\left\{X_{j}\right\}$ the sequence of sets in ( $\left.\mathrm{II}^{\prime}\right)$. Then from Birkhoff's ergodic theorem it follows that the limit function $\widetilde{\varphi}_{A}$ of the means of $\phi_{A}$ is

7) The proof of this part is due to Ryll-Nardzewski [4]. 
(8)

$$
\int_{x} \widetilde{\varphi}_{A}(x) d \mu=\mu(A)
$$

defined almost everywhere $(\mu)$ and hence almost everywhere $(m)$, and that Since $\mu$ is equivalent to $m$ and $m(A)>0$, it follows $\mu(A)>0$. Let $B$ be the set $\left\{x ; \widetilde{\phi}_{A}(x)>0\right\}$, then, from $\mu(A)>0$ and (8), it follows $\mu(B)>0$. Hence we can choose a set $X_{j_{0}} \in\left\{X_{i}\right\}$ such that $\mu\left(X_{j_{0}} \cap B\right)>0$. Since $m$ is equivalent to $\mu$, it holds $m\left(X_{j_{0}} \cap B\right)>0$. Thus we have

$$
\begin{aligned}
& \sup _{j} \lim _{n} \sup \frac{1}{n} \sum_{i=0}^{n-1} m\left(X_{i} \cap T^{-i} A\right) \geqq \lim \sup _{n} \frac{1}{n} \sum_{i=0}^{n-1} m\left(X_{i_{0}} \cap T^{-i} A\right) \\
& =\lim _{n} \sup _{n} \frac{1}{n} \sum_{i=0}^{n-1} \int_{x_{j_{0}}} \phi_{t}\left(T^{i} x\right) d m \geqq \int_{x_{j_{0}}} \lim \inf _{n} \frac{1}{n} \sum_{i=0}^{n-1} \phi_{A}\left(T^{i} x\right) d m \\
& =\int_{x_{j_{0}}} \widetilde{\varphi}_{A}(x) d m>0 .
\end{aligned}
$$

Combining $\left(\mathrm{II}^{\prime}\right)$ and the above inequality we get (II).

From Theorem 2 and the fact proved above, it follows that the assu. mption of the theorem cannot be omitted (cf. Example 1 in $\S 3$ ).

THEOREM 4. If $X$ is the union of countable invariant subsets of finite measure, then the statements (II), (II') and $(B)$ are equivalent to each other.

The assumption of the theorem cannot be omitted.

Proof. Theorem 2 shows that (II) implies $(B)$ and $(B)$ implies (II'), so that for the purpose it is sufficient to prove that (II') implies (II).

From the assumption there exist the invariant sets $Y$;'s such that

$$
X=\bigcup_{j=1}^{\infty} Y_{j}, \quad Y_{i} \cap Y_{j}=0(i \neq j), 0<m\left(Y_{j}\right)<\infty(j=12, \ldots) .
$$

If we define the set function $\beta^{*}$ as in the proof of Theorem 2 , then it is easy to see that, for each $j, \beta^{k}$ is a finite invariant measure on $\mathfrak{B}_{Y_{j}}$ and is equivalent to $m$ as the measure on $\mathfrak{B} Y_{j}$.

Let us now put for any set $A$

$$
\mu(A)=\sum_{j=1}^{\infty} \beta^{*}\left(A \cap Y_{j}\right) / 2^{j} \beta^{*}\left(Y_{3}\right)
$$

Then it is clear that $\mu$ is a finite invariant measure on $\mathfrak{B}$ equivalent to $m$. Hence we obtain the conclusion by Theorem 3 .

It follows, from Theorem 2 and the fact proved above, that the assumption of the theorem cannot be omitted (cf. Example 1 in $\S 3$ ).

REMARK. Theorem 3 is essentially equivalent to Theorem 4.

In the Proof of Theorem 4 we have shown that the assumption of Theorem 3 follows from the assumption of Theorem 4 and the statement (II'), so tiat $f$ or the present purpose it is sufficient to show that the assu- 
mption of Theorem 3 and the statement $(B)$ imply the assumption of Theorem 4.

Let $\mathfrak{B}_{0}$ denote the class such that sets of $\mathfrak{B}_{0}$ are the invariant sets of finite positive measure and are mutually equivalent. Since $m$ is $\sigma$-finite, the class $\mathfrak{B}_{\mathrm{v}}$ is at most countable. Let us denote by $Y$ the union of all sets of $\mathfrak{B}_{0}$, then the set $X-Y$ must be a set of measure zero or an invariant set which has no invariant subsets of finite positive measure.

Now let us suppose that $X-Y$ is not the set of measure zero. Then there is a set $A$ such that

$$
A \subset X-Y, 0<m(A)<\infty \text {. }
$$

Since $\phi_{A} \in L(X, \mathfrak{B}, m)$ and $(B)$ holds, the limit $\widetilde{\varphi}_{1}$ of the means of $\phi_{A}$ is defined almost everywhere $(m)$ and $\widetilde{\varphi}_{A} \in L(X, \mathfrak{R}, m)$.

On the other hand, let $\mu$ be a finite invariant measure equivalent to $m$, then from Birkhoff's ergodic theorem it follows that $\widetilde{\phi}_{A}$ is dsfined almost everywhere $(\mu)$ and that

$$
\int_{X} \widetilde{\varphi}_{A}(x) d \mu=\mu(A) .
$$

From $m(A)>0$ we get $\mu(A)>0$. Hence, if we put $B=\left\{x ; \widetilde{\boldsymbol{\phi}}_{A}(x)>0\right\}$, then from (9) and $\mu(A)>0$ it follows $\mu(B)>0$, so that $m(B)>0$. Then there exists a positive number $\varepsilon$ such that the set $\left\{x ; \widetilde{\phi}_{A}(x)>\varepsilon\right\}$ is of positive measure. Since $\widetilde{\varphi}_{A}$ is the invariant function and $\widetilde{\phi}_{A} \in L(X, \mathfrak{B}, m)$, the set $\{x$, $\left.\widetilde{\boldsymbol{\phi}}_{A}(x)>\varepsilon\right\}$ is an invariant set of finite positive measure. This contradicts the assumption for the set $X-Y$. Hence $X-Y$ is the set of measure zero.

Thus we can conclude that $X$ is the union of countable invariant subsets of finite measure.

3. Counter examples. We shall now show by example that, in case $m$ is not finite, $(B)$ does not necessarily imply (II) and that (II') does not necessarily imply $(B)$.

EXAMPLE 1. We shall start from the measure space $(X, \mathfrak{B}, \mu)$ and the transformation $T$ constructed by P. R. Halmos [2; pp. 743-744].

Let us define the collection of the linear intervals $J_{n, k}$ 's in the $(s, t)$-plane by

$$
J_{n, k}=\left\{(s, k) ; \quad \frac{1}{2^{n+1}} \leqq s<\frac{1}{2^{n}}\right\}\left(\begin{array}{l}
k=0,1, \ldots, 2^{n+1}-1 ; \\
n=0,1,2, \ldots
\end{array}\right) .
$$

Let $(X, \mathfrak{B}, \mu)$ be the measure space such that $X$ is the union of all $J_{n, k}$ 's, $\mathfrak{B}$ is the class of the Lebesgue measurable subsets of $X$, and $\mu$ is the ordinary linear Lebesgue measure on $\mathfrak{B}$. Let $T_{0}$ be any one to one, measurable, measure-preserving (with respect to $\mu$ ), and ergodic transformation of $\bigcup_{n=0}^{\infty} J_{n, 0}$ onto itself (for example, the transformation $T_{0}$ is defined by 


$$
T_{0}(s, 0)=(\{s+\theta\}, 0), \quad(s, 0) \in \bigcup_{n=0}^{\infty} J_{n, 0},
$$

where $\theta$ is a fixed irrational number and $\{s+\theta\}$ denotes the fractional part of $s+\theta$ ). Further, let us define a transformation $T$ by

$$
\begin{aligned}
& T(s, t)=(s, t+1), \text { if }(s, t) \in J_{n, k}\left(\begin{array}{l}
k=0,1, \ldots, 2^{n+1}-2 ; \\
n=0,1,2, \ldots
\end{array}\right) \\
& =T_{0}(s, 0) \text {, if }(s, t) \in J_{n, 2}{ }^{n+1}-1(n=0,1,2, \ldots) .
\end{aligned}
$$

Then it can be proved that $T$ is a one to one, measurable, measure-preserving (with respect to $\mu$ ), and ergodic transformation of $X$ onto itself (see [2]).

In the following, by $x$ we denote the point $(s, t)$ of $X$ simply.

From Birkhoff's ergodic theorem it follows that for any function $f \in$ $L(X, \mathfrak{B}, \mu)$ the limit function $\tilde{f}$ of the means of $f$ is defined almost everywhere $(\mu)$ and $\widetilde{f} \in L(X, \mathfrak{B}, \mu)$.

Since $\widetilde{f}$ is an invariant function and $T$ is ergodic, the function $\widetilde{f}(x)$ is constant almost everywhere $(\mu)$, so that $\widetilde{f} \in L(X, \mathfrak{B}, \mu)$ implies that $\widetilde{f}(x)$ vanishes almost everywhere $(\mu)$. Hence, for any sets $Y$ and $A$ of finite measure, we have

$$
\begin{aligned}
\lim \sup _{n} & \frac{1}{n} \sum_{i=0}^{n-1} \mu\left(Y \cap T^{-i} A\right)=\lim \sup _{n} \frac{1}{n} \sum_{i=0}^{n-1} \int_{Y} \Phi_{A}\left(T^{i} x\right) d \mu \\
& \leqq \int_{Y} \lim \sup _{n} \frac{1}{n} \sum_{i=0}^{n-1} \phi_{A}\left(T^{i} x\right) d \mu=\int_{Y} \widetilde{\varphi}_{A}(x) d \mu=0
\end{aligned}
$$

so that the statement (II) does not holds.

On the other hand, since $\mu$ is invariant under $T$, the statement $(B)$ is the immediate consequence of Birkhoff's ergodic theorem.

Thus we conclude that $(B)$ does not necessarily imply (II) (see Theorem 2).

Next we define a new measure $m$ on $\mathfrak{B}$ as follows :

$$
\begin{aligned}
m(A) & =\mu(A) /\left[2(n+1)^{2}-1\right], \text { if } A \subset J_{n, 2}{ }^{n+1}-1(n=0,1, \ldots), \\
& =\mu(A), \quad \text { if } A \subset J_{n, k}\left(\begin{array}{l}
k=0,1, \ldots, 2^{n+1}-2 ; \\
n=0,1,2, \ldots
\end{array}\right) .
\end{aligned}
$$

Then it is obvious that $m$ is a $\sigma$-finite (but not finite) measure equivalent to $\mu$, and $T$ is measurable, non-singular (with respect to $m$ ) and ergodic. We shall now show that the measure space $(X, \mathfrak{B}, m)$ and the transformation $T$ have the following properties :

(i) the statement $(B)$ does not hold;

(ii) the statement (II') holds.

Proof of (i): Let us put

$f(x)=2^{n+1}\left[2(n+1)^{2}-1\right] / 2(n+1)^{2}$, if $x \in J_{n, 2}{ }^{n+1}-1(n=0,1,2, \cdots)$, 


$$
=2^{n+1} / 2(n+1)^{2}\left(2^{n+1}-1\right), \text { if } x \in J_{n, k}\left(\begin{array}{l}
k=0,1, \ldots, 2^{n+1}-2 ; \\
n=0,1,2, \ldots
\end{array}\right) .
$$

Then we have

$$
\begin{aligned}
\int_{X} f(x) d m= & \sum_{n=0}^{\infty} \int_{J_{n, 2}{ }^{n+1}-1} f(x) d m+\sum_{n=0}^{\infty} \sum_{k=0}^{2^{n+1}-2} \int_{J_{n, k}} f(x) d m \\
= & \sum_{n=0}^{\infty}\left(\frac{2^{n+1}\left[2(n+1)^{2}-1\right]}{2(n+1)^{2}}\right) \cdot\left(\frac{1}{2^{n+1}} \cdot \frac{1}{2(n+1)^{2}-1}\right) \\
& \quad+\sum_{n=0}^{\infty} \sum_{k=0}^{2^{n+1}-2}\left(\frac{2^{n+1}}{2(n+1)^{2}\left(2^{n+1}-1\right)}\right) \cdot\left(\frac{1}{2^{n+1}}\right) \\
= & \sum_{n=0}^{\infty} \frac{1}{(n+1)^{2}}<\infty,
\end{aligned}
$$

so that $f \in L(X, \mathfrak{B}, m)$.

On the other hand, it holds that for each $n$

$$
\begin{gathered}
f(x)+f(T x)+\ldots+f\left(T^{2^{n+1}-1} x\right) \\
=\frac{2^{n+1}}{\left.2(n+1)^{2\left(2^{n+1}-1\right)}+\frac{2^{n+1}}{2(n+1)^{2}\left(2^{n+1}-1\right)}+\cdots+2(\overline{n+1})^{2\left(2^{n+1}\right.}-1\right)} \\
\left(2^{n+1}-1\right) \text { terms } \\
+\frac{2^{n+1}\left[2(n+1)^{2}-1\right]}{2(n+1)^{2}}=2^{n+1}, \quad x \in J_{n, 0},
\end{gathered}
$$

and further to any point $x$ of $X$ there corresponds a positive integer $p(x)$ such that

$$
T^{p(x)} x \in \bigcup_{n=0}^{\infty} J_{n, 0} .
$$

Hence we get for any $x$ of $X$

$$
\lim \sup _{n} \frac{1}{n} \sum_{i=0}^{n-1} f\left(T^{i} x\right)=1 .
$$

If we suppose that $(B)$ holds, then we have

$$
\widetilde{f}(x)=\lim _{n} \frac{1}{n} \sum_{i=0}^{n-1} f\left(T^{\prime} x\right)=1
$$

almost everywhere $(m)$ and $\widetilde{f} \in L(X, \mathfrak{B}, m)$. This contradicts the fact that $m$ is not finite. Hence $(B)$ does not hold.

Proof of (ii): It is easy to see that for any set $A$ of finite measure

$$
m(A) \leqq \mu(A)<\infty .
$$

By (1) and (2) we get that for any sets $A$ and $Y$ of finite measure

$$
\lim \sup _{n} \frac{1}{n} \sum_{i=0}^{n-1} m\left(Y \cap T^{-i} A\right)=0 \text {. }
$$


Since the inequality in $\left(\mathrm{II}^{\prime}\right)$ holds evidently for any set $A$ of infinite measure, the statement $\left(\mathrm{II}^{\prime}\right)$ holds.

Thus we conclude that $\left(\mathrm{II}^{\prime}\right)$ does not necessarily imply $(B)$ (see Theorem 2).

In the connection to Theorem 3 and 4 we note that the following properties can be easily shown:

(iii) there exists no finite invariant measure equivalent to $m$;

(iv) $X$ has no invariant subset of finite measure.

Finally we shall show by example that, in case $m$ is not finite, $(B)$ does not necessarily imply $\left(\mathrm{I}^{\prime}\right)$.

Example 2. We define $X, \mathfrak{B}, \mu, T$ and $J_{n, k}\left(k=0,1, \ldots 2^{n+1}-1 ; n=0\right.$, $1,2, \ldots)$ as in Example 1. We introduce a new $\sigma$-finite measure $\boldsymbol{m}$ on $\mathfrak{B}$ such that $m$ is equivalent to $\mu$, and for any set $A$

$$
m\left(A \cap J_{n, k}\right)=\mu\left(A \cap J_{n, k}\right)\left(\begin{array}{l}
k=1,2, \ldots, 2^{n+1}-1 ; \\
n=0,1,2, \ldots
\end{array}\right)
$$

and

$$
m\left(J_{n, 0}\right)=\infty, \quad m\left(A \cap J_{n, 0}\right) \geqq \mu\left(A \cap J_{n, 0}\right)(n=0,1,2, \ldots) .
$$

In fact, it is easy to construct such measure.

Let us suppose $f \in L(X, \mathfrak{B}, m)$, then by (3) and (4) we get $f \in L(X, \mathfrak{B}, \mu)$. Hence we get similarly as in Example 1 that the limit function $\widetilde{f}$ of the means of $f$ is defined almost everywhere $(m)$ and $\widetilde{f}(x)$ vanishes almost everywhere $(m)$. Thus the statement $(B)$ holds.

On the other hand, if we put

$$
A=\bigcup_{n=0}^{\infty} J_{n, 2}{ }^{n+1}-1
$$

we have

$$
m(A)=\mu(A)=1 .
$$

Then from (4) it follows that for each $n$

$$
\begin{aligned}
& \frac{1}{2^{n+1}} \sum_{i=0}^{2^{n+1}-1} m\left(T^{-i} A\right) \geqq \frac{1}{2^{n+1}} \sum_{i=0}^{2^{n+1}-1} m\left(T^{-i} J_{n, 2}{ }^{n+1}-1\right) \\
& \geqq \frac{1}{2^{n+1}} m\left(T^{-\left(2^{n+1}-1\right)} J_{n, 2}{ }^{n+1}-1\right)=\frac{1}{2^{n+1}} m\left(J_{n, 0}\right)=\infty
\end{aligned}
$$

so that

$$
\lim \sup _{n} \frac{1}{n} \sum_{i=0}^{n-1} m\left(T^{-i} A\right)=\infty .
$$

By (5) and (6) we have that $\left(I^{\prime}\right)$ does not hold.

Thus we conclude that $(B)$ does not necessarily imply $\left(I^{\prime}\right)$ and then $(I)$ (see Theorem 1).

Finally I have to express my cordial thanks to Mr.S. Yano who gave me valuable remarks and advices. 


\section{SUPPLEMENT}

The results of the present paper were sketched in the "preliminary report, S. Tsurumi, On ergodic theorems, Proc. Japan Acad., 30(1954) pp. 331-334 in which the sentence misinserted in lines $16-17$ of page 333 should be omitted.

\section{REFERENCES}

[1] N. DunFord and D. S. Miller, On the ergodic theorems Trans. Amer. Math. Soc., 60(1946), pp. 538-549.

[2] P. R. HALmOS, Invariant measures, Ann. of Math. 48(1947), pp. 735-754.

[3] F. RIESz, On a recent generalisation of G.D. Birkhoff's ergodic theorem, Acta de Szeged, 11(1946-1948), pp. 193-200.

[4] C. RYLL-NARDZEWSKI, On the ergodic theorems (I) (Generalized ergodic theorems), Studia Math., 12(1951), pp. 65-73.

Mathematical Institute, TOKyo Metropolitan University. 